\title{
Incorporating the environmental dimension in the assessment of automated warehouses
}

In today's competitive context, the paradigm of sustainable development is becoming more and more significant, also in warehousing. Managers are progressively considering not only purely economic aspects but also environmental concerns. Despite such consciousness, the selection of automated warehousing solutions has been mainly based on operational and economic performance in both practice and theory so far, whereas energy consumption and environmental performance have not been adequately taken into account. To fill this lack a model is proposed to evaluate the energy consumption and environmental impact of automated warehousing solutions. The model has been used to investigate whether the selection of automated solutions changes varying the dimensions involved in the analysis (i.e. only economic, only environmental or both). The analysis has been performed considering autonomous vehicle storage and retrieval systems (AVS/RSs) and its natural alternative, i.e. automated storage and retrieval systems (AS/RSs). Results confirm the importance of considering both dimensions in the assessment of automated warehouses, as depending on the scenario the technology selection shifts from AS/RS to AVS/RS when considering not only the economic but also the environmental impact. Additionally, this study provides new insights on the suitability areas of AVS/RSs.

Keywords: automated warehouses; material handling; sustainable development; energy efficiency

\section{Introduction}

In recent years, a rising attention has been paid to supply chain sustainability, both by organisations (Mahler 2007) and researchers (Seuring and Müller 2008). Increasing social awareness together with the new regulations for carbon and waste management are forcing enterprises to reconsider their supply chains with respect to economic, social and environmental objectives (Chaabane, Ramudhin, and Paquet 2011). In this context, exploring the impact of environmentally sustainable initiatives on 
company's supply chain has become very important (Barber, Beach, and Zolkiewski 2012), especially with regard to logistics activities. In terms of logistics, the focus has been on understanding and mitigating the impact of transportation activities (e.g. Ballot and Fontane, 2010; Tan, Ahmed, and Sundaram 2010), whereas the issue of "warehouse sustainability" seems to be understudied, although in literature it has been recognised as one of the most interesting topics for future research (McKinnon, Browne, and Whiteing 2012). A number of contributions have been developed offering approaches towards more sustainable warehouses, for instance related to green building practices (Rizzo 2006), the use of green energy sources (e.g. cogeneration plants) and operational strategies (e.g. travel distance optimisation, storage assignment policies), and the adoption of energy-efficient material handling equipment (Colicchia, Melacini, and Perotti 2011). However, to the best of the author's knowledge, no studies have been conducted on the estimation of warehouse environmental impact so far. This lack is particularly significant for automated solutions, as they can potentially further increase the need of energy (MHIA 2009).

At the same time, a wide range of solutions with a high automation level have been progressively introduced (Dallari, Marchet, and Melacini 2009), some of which bringing the promise of potential improvements in both energy consumption and throughput capacity compared to other automated systems. According to material handling providers, one such significant example lies in the autonomous vehicle storage and retrieval system (AVS/RS). AVS/RS is a widely-used automated material handling technology where unit loads are handled by vehicles along the horizontal dimension within the storage racks and by lift along the vertical dimension (Ekren and Heragu 2012; Marchet et al. 2012). 
In this context, the estimation of warehouse environmental impact could improve and enrich the assessment of automated warehousing solutions in the technology selection problem. Today, this selection is mainly based on both the system performance and the economic dimension, without taking into account environmental concerns (Meneghetti and Monti 2013). This may lead to the selection of a suitable warehousing system in terms of performance and costs, although it may not necessarily be satisfactory from an environmental sustainability perspective.

Moreover, data on warehouse environmental impact allows investigating whether the existing trade-off between the economic and environmental dimensions in the broader context of logistics (Quariguasi Frota Neto et al. 2009) is also applicable to warehousing solutions.

Based on this premise, the objective of this paper is (i) to incorporate the environmental perspective in the assessment of warehousing solutions for a new warehouse, and (ii) to investigate the trade-off between the environmental and economic dimensions when selecting automated warehousing technologies (i.e. to investigate whether the selection of automated solutions changes varying the considered dimensions). To this extent, a model for the assessment of the energy consumption and environmental impact of warehousing automated systems is developed. The model has been used to investigate whether and how the assessment and selection of automated warehousing systems are affected by the inclusion of the environmental perspective. Specifically, the AVS/RS technology and its natural alternative, i.e. automated storage and retrieval system (AS/RS), have been examined.

The remainder of the paper is organised as follows. The previous literature on the assessment of automated warehousing systems and the evaluation of environmental aspects is discussed in Section 2. The descriptions of the two automated solutions 
examined in this paper (i.e. AS/RS and AVS/RS technologies) are reported in Section 3, whereas the model for assessing the environmental performance of automated systems is proposed in Section 4. In Section 5, the model is used to explore the trade-off between the economic and environmental dimensions by evaluating the performance of AS/RSs and AVS/RSs. In particular, a threefold approach has been adopted: (i) taking into account only the economic dimension, (ii) looking only at the environmental dimension, and (iii) considering the economic and environmental dimensions simultaneously. Conclusions, limitations and suggestions for future research are presented in Section 6.

\section{Literature review}

The literature review has been structured into two subsections. First of all, we took into account how the assessment of warehousing solutions has been performed previously in terms of both economic and environmental performance, with a focus on the comparison between AS/RS and AVS/RS technologies. Secondly, since the environmental perspective has seldom been considered when evaluating warehousing solutions, we examined how the environmental perspective has been incorporated in performance assessment within a broader scope of research, i.e. supply chain.

\subsection{Economic and environmental performance assessment of automated warehouses}

In the warehousing literature, the assessment of warehousing technologies traditionally belongs to the branch of research on the 'equipment selection problem'. As underlined by Gua, Goetschalckx, and McGinnis (2010), research in this area addresses warehouse automation level and the type of storage and material handling systems to be adopted based on the comparison among several possible alternatives. 
Overall, in the extant literature, the most widespread approach in the evaluation of automated material handling solutions is mainly based on operational and economic performance measures, such as travel time per request, number of requests handled per time period, waiting times for products to be stored or retrieved, and throughput time (Roodbergen and Vis 2009; Gua, Goetschalckx, and McGinnis 2010), whereas the assessment of the energy consumption has been often disregarded so far.

As far as the economic performance is concerned, a number of studies considered equipment and labour cost to compare different warehousing solutions or operational policies. For example, Malmborg (1996) included inventory management, space allocation between reserve and storage area, and storage area layout in a single integrated model that takes into account a number of major cost parameters associated with such distribution system activities. To select between batch and zone order picking strategies, Parikh and Meller (2008) developed a cost model including the costs of pickers, equipment, imbalance, sorting, and packers. Marchet, Melacini, and Perotti (2011) performed a comparison between construction costs (i.e. sorting and accumulation lanes) and operational costs (i.e. cost of pickers) to compare the picking efficiency for a pick-and-sort system in different operating environments.

Focusing on the evaluation of the economic comparison between AS/RS and AVS/RS technologies, the first studies addressing this issue were performed by Kuo, Krishnamurthy, and Malmborg (2007), and Fukunari and Malmborg (2008). Among the different solution types based on the AVS/RS technology, the authors considered a system for pallet handling in which each vehicle can access any tier by using the lift. Fifteen problem scenarios were considered, with storage capacities ranging from 10,000 to 30,000 storage positions, and transaction demand levels ranging from 100 to 300 requests per hour. For each scenario, a comparison was made between the lowest-cost 
AVS/RS and AS/RS configurations, where vehicle or crane utilisation was below $90 \%$. Equipment costs were assessed, namely for vehicles and lifts in AVS/RS and cranes and accumulation conveyors in AS/RS. Similarly, Ekren and Heragu (2012) compared AS/RS and AVS/RS performance varying their storage capacity and transaction demand level. All of these studies showed that AVS/RS for pallets appears to be most suitable in case of warehouses characterised by low throughput volume to storage capacity ratios.

As far as the environmental performance is concerned, Meneghetti and Monti (2013) proposed a model for the evaluation of energy consumption to compare different storage location and dwell point strategies from an energy-efficiency viewpoint in an AS/RS. The model is mainly based on the estimation of the mechanical energy provided by motors through the integral of the power (equal to the product of the torque times the angular speed) over time. The model has not been validated and it does not allow estimating the energy consumption as a function of the warehouse characteristics (i.e. equipment features and rack configuration). To the best of the authors' knowledge, this is the only contribution addressing the problem of the energy consumption in automated warehouses.

In summary, a widely acknowledged holistic model for the estimation of the energy consumption of automated warehousing solutions is still lacking, and their environmental performance has not been adequately evaluated yet.

\subsection{Approaches for evaluating the trade-off between economic and environmental perspectives in supply chains}

A company that aims to move towards sustainability has to consider and balance costs as well as environmental concerns (Gimenez, Sierra, and Rodon 2012). In the supply chain literature, to-date, economic and environmental performance have been examined either separately or jointly by evaluating the potential trade-off between these two 
different perspectives. The exploration of this trade-off has led to the examination of efficient frontiers for business and the environment (Huppes and Ishikawa 2005; Bloemhof-Ruuward, Krikke, and Van Wassenhove 2004). As illustrated by Quariguasi Frota Neto et al. (2009), the rationale is to identify a set of solutions for which it is not possible to decrease environmental impact without increasing costs.

Different approaches have been developed to evaluate the trade-off between economic and environmental perspectives. A first option consists in relegating the choice of the solution to the management based on the dimension (i.e. economic or environmental) considered as more important (Langella and Zanoni 2011). A second method is to adopt multi-objective models (Ramudhin, Chaabane, and Paquet 2009), whereas a third alternative lies in adopting the same metric for both the economic and environmental aspects, i.e. by monetising the environmental impact. As illustrated by Pearce (2002), this latter approach has opened a debate. On the one hand, it seems particularly valuable as it easily allows an assessment to be made strictly based on costs. On the other hand, concerns have been raised that it may be inappropriate, due to difficulties with the monetisation of environmental impact.

In this regard, different methodologies have been developed for monetising the environmental impact. Focusing on the impact of $\mathrm{CO}_{2}$ emissions, four main approaches have been identified: marginal abatement cost (Klepper and Peterson 2006), shadow price of carbon (Price, Thornton, and Nelson 2007), trading price (Ramudhin, Chaabane, and Paquet 2009) and social cost of carbon (Nordhaus 2011). These methodologies are based on a number of assumptions (e.g. on the social discount rate and the expected increase in the global average temperature), which results in a considerable variability in the $\mathrm{CO}_{2}$ unit cost. In their recent study, Johnson and Hope (2012) performed a sensitivity analysis on the parameters involved in the monetisation 
of the environmental impact. Their results suggested that the value of Social Cost of Carbon ranges from 16 dollars to 758 dollars per $\mathrm{tCO}_{2}$ depending on the assumptions made.

In summary, several studies in the supply chain literature took into account the environmental performance, especially through the analysis of the carbon footprint, and approaches for analysing the trade-off between environmental and economic dimensions have been provided. Among them, the monetisation of the environmental impact seems to be the one that has received more attention so far.

\section{Description of automated warehousing systems}

This section provides a description of the technologies examined (i.e. AS/RS and AVS/RS), as well as the main assumptions on which the proposed model is based in order to evaluate the environmental impact. The main notations used in the remainder of the paper are also introduced.

The foremost difference between the two considered systems lies in the devices used for unit load handling. In an AS/RS, storage cranes that move simultaneously along vertical and horizontal dimensions handle unit loads. Conversely, in an AVS/RS unit loads are handled by vehicles that move horizontally along rails within the storage racks, whereas vertical movement is provided by lifts mounted along the rack periphery (Malmborg 2002). The two technologies can be used to handle both pallets and product totes. As the current trend is a progressive reduction in order size (Baker and Canessa 2009), with an increase in tote retrieval as opposed to pallets, the analysis focuses on the use of totes as the handling unit. More specifically, as far as the two above-described technologies are concerned, the following widely adopted configurations are examined in this study: 
- AS/RS characterised by aisle-captive storage cranes;

- AVS/RS in which each aisle has one lift and one vehicle dedicated to each tier (Figures 1 and 2). As shown on the figures, the first position at each side of the storage aisle in all tiers serves as a buffer and is used to transfer unit loads from vehicles to lifts. One buffer (called buffer out) handles the unit loads that have been retrieved, whereas the other one (called buffer in), located in the other side of the storage aisle, handles the unit loads to be stored. This choice allows the mutual independence of the vehicles and lifts.

\section{XXXXXXXXXXXXXXXXXXXXXXXXXXXXXXXXXXXXXXXXXXXXXXXXX}

Take in Figures 1 and 2

\section{XXXXXXXXXXXXXXXXXXXXXXXXXXXXXXXXXXXXXXXXXXXXXXXXX}

In both AS/RS and AVS/RS, single-deep, double-sided storage racks are used, and the input/output $(\mathrm{I} / \mathrm{O})$ points are located at the heads of each aisle. A random storage policy is used to allocate products to storage positions. The assumed dwell point is the I/O point for cranes in an AS/RS, whereas in an AVS/RS the dwell point consists in the buffer positions and the I/O points for vehicles and lifts, respectively. Only single command cycles are considered, as performing a high percentage of dual command cycles in an AVS/RS is rather difficult (Zhang et al. 2009). Moreover, both storage and retrieval cycles are served by vehicles and lifts according to the FCFS (first-come-firstserve) policy.

The main notations used in the remainder of the paper are reported in Table 1.

XXXXXXXXXXXXXXXXXXXXXXXXXXXXXXXXXXXXXXXXXXXXXXXXX

Take in Table 1

XXXXXXXXXXXXXXXXXXXXXXXXXXXXXXXXXXXXXXXXXXXXXXXXX 


\section{Environmental impact of automated warehouses: an assessment model}

The environmental impact of an automated warehousing solution is mainly a function of the indirect $\mathrm{CO}_{2}$ emissions related to the energy consumption. Carbon dioxide is the oxidised form of carbon and it is the major greenhouse gas implicated in projections of global warming (Pearce 2002). The estimate of carbon emissions depends on the warehouse energy source(s). In case the energy is produced in-house (e.g. by means of photovoltaic panels), the resulting environmental impact is negligible. Conversely, when energy is purchased externally, the impact depends on the type(s) of energy source(s) used by the provider.

The assessment of the environmental impact $\left(I^{e n v}\right)$ of an automated warehousing solution involves the evaluation of the energy consumption $(E)$ and the definition of the factor $(\alpha)$ that converts the energy consumption into indirect $\mathrm{CO}_{2}$ emissions. In turn, the energy consumption $E$ in the reserve area mainly consists of two components: energy required to maintain a target temperature $\left(E^{T}\right)$ and energy required for handling operations $\left(E^{H}\right)$.

$$
I^{e n v}=E * \alpha=\left(E^{T}+E^{H}\right) * \alpha
$$

$E^{T}$ is a function of the heat exchange through the walls. In turn, heat exchange through the walls may be viewed as a function of a number of factors, such as the required temperature in the reserve area, the external temperature, the overall area of the side walls, and their thermal insulation that is related to their thickness as well as the construction material(s). Specific normative can be used to obtain $E^{T}$ (e.g. UNI/TS 11300).

With reference to the second component $\left(E^{H}\right)$, a widely acknowledged approach for estimating energy consumption as a function of both rack configuration and 
characteristics of devices for the unit load movements is still lacking (please refer to the literature review section). Therefore, we propose an energy model aiming at filling this gap, according to which $E^{H}$ may be calculated as the product of the total number of cycles in a year and the average energy consumption per cycle $\left(E_{c}^{H}\right)$.

The estimation of the average energy consumption per cycle $\left(E_{c}^{H}\right)$ can be calculated as the weighted average of the energy consumption of a single command cycle for each storage position $i\left(E_{i}^{H}\right)$ :

$$
E_{c}^{H}=\sum_{i=1}^{n} p_{i} * E_{i}^{H}
$$

where $n$ is the total number of storage positions and $p_{i}$ is the probability that the storage position $i$ is involved in a cycle characterised by a given storage tier $t$ and storage column $c$.

In both $\mathrm{AS} / \mathrm{RS}$ and $\mathrm{AVS} / \mathrm{RS}$, the energy required to reach a given storage position $\left(E_{i}^{H}\right)$ is the sum of the energy consumption for movements along the $z-\left(E_{i}^{Z}\right)$ and $y$-axes $\left(E_{i}^{y}\right)$. The two motors that allow the crane to move along the $z$ - and $y$-axes simultaneously are independent in an AS/RS, whereas the movements along the $z$ - and $y$-axes are carried out by different devices (i.e. lifts and vehicles, respectively) in an AVS/RS. The values of $E_{i}^{z}$ and $E_{i}^{y}$ are strictly related to the activities involved in completing a cycle along the two axes. Under the dwell point policy described in Section 3, the following movement elements required for both AS/RS and AVS/RS can be identified:

- Movement A, along the $z$-axis from the I/O point to the destination tier $t$

- Movement B, along the $z$-axis from the destination tier $t$ to $\mathrm{I} / \mathrm{O}$ point

- Movement $\mathrm{C}$, along the $y$-axis from the buffer to the destination column $c$ 
- Movement $\mathrm{D}$, along the $y$-axis from the destination column $c$ to the buffer

Note that the aforementioned movement elements are valid for both types of single command cycle (i.e. storage and retrieval). Therefore, $E_{i}^{H}$ is given by the following expression:

$$
E_{i}^{H}=E_{i}^{Z}+E_{i}^{y}=E_{i}^{A}+E_{i}^{B}+E_{i}^{C}+E_{i}^{D}
$$

Before describing the energy consumption of each movement element in detail, it is important to note the main assumptions used in the model:

- In order to model explicitly the relationship between the energy consumption and the features of the automated solution (i.e. rack configuration and equipment characteristics), the mechanical energy required is divided by an efficiency factor $\eta$ that considers the ratio between the shaft power out to the electrical power into the motor, as well as the kinematics of the devices.

- A trapezium velocity profile was used. This entails an acceleration warm up, a phase in which the velocity is constant and set at its maximum value, and a deceleration warm up. When the travel distance is not far enough for the maximum velocity to be reached, a triangular velocity profile, which includes only the acceleration and deceleration phases, is used.

- To be more consistent with recent applications that reduce energy consumption, it was assumed that cranes in an AS/RS are controlled in such a way that horizontal and vertical movements end simultaneously. In this situation, the travel time associated with the fastest movement is decreased by applying a velocity value lower than the nominal one. 
- The energy consumed to load/unload a tote to or from storage and retrieval (S/R) devices was not included in the model due to its marginal impact on the overall energy used to complete a cycle and because it does not depend on the rack configuration.

- It was assumed that the motor does not operate as a generator. This means that, for example, the energy produced during the descending phase is not reused but is fully dissipated.

Given the main assumptions listed above and the notations reported in Table 1, the energy consumption associated with each element movement is estimated as follows:

- The energy consumption $E_{i}^{A}$ associated with movement elements A (i.e. movement along the $z$-axis from the $\mathrm{I} / \mathrm{O}$ point to the destination tier $t$ ) is expressed by the ratio of the change in potential energy $\Delta U_{i}$ to the motor efficiency $\eta$ :

$$
E_{i}^{A}=\frac{\Delta U_{i}}{\eta}=\frac{m_{1} g h_{i}}{\eta}
$$

where $m_{1}$ is the mass of the equipment (i.e. the mass of the crane component that moves vertically in an AS/RS, $m_{1}^{A S / R S}$, or the mass of the lift in an AVS/RS, $m_{1}^{A V S / R S}$ ) to which the mass of the load $m_{l}$ must be added for storage cycles; $g$ is the gravitational acceleration; and $h_{i}$ is the height associated with the destination tier $t$.

- The energy consumption $E_{i}^{B}$ associated with movement elements B (i.e. movement along the $z$-axis from the destination tier $t$ to the $\mathrm{I} / \mathrm{O}$ point) is null 
due to the aforementioned hypothesis that the motor does not operate as a generator.

- The energy consumption associated with each of the two movement elements C and $\mathrm{D}$ (i.e. movements along the $y$-axis from the buffer to the destination column $c$ and from the destination column $c$ to the buffer, respectively), $E_{i}^{C}$ and $E_{i}^{D}$, are related by the sum of the kinetic energy $K_{i}$ during the acceleration phases and the friction energy loss $F E_{i}$ during the acceleration warm up and the constant velocity phase. More specifically, $E_{i}^{C}$ and $E_{i}^{D}$ are expressed as follows:

$$
E_{i}^{C}=E_{i}^{D}=\frac{K_{i}+F E_{i}}{\eta}=\frac{\frac{1}{2} m_{2} v_{i}^{2}+\mu m_{2} g \Delta s_{i}}{\eta}
$$

where $m_{2}$ is the mass of the equipment (i.e. the mass of the crane component that moves horizontally in an AS/RS, $m_{2}^{A S / R S}$, or the mass of the vehicle in an AVS/RS, $m_{2}^{A V S / R S}$ ) to which the mass of the load $m_{l}$ must be added for storage or retrieval cycles when calculating $E_{i}^{C}$ and $E_{i}^{D}$, respectively; $v_{i}$ is the maximum velocity reached; $\mu$ is the coefficient of friction; $g$ is the gravitational acceleration; and $\Delta s_{i}$ is the travel distance covered during the acceleration warm up and the constant velocity phase.

In summary, assuming that the system performs storage and retrieval cycles, the average energy consumption per cycle $E_{i}^{H}$ can be calculated using Equation (6):

$$
\begin{gathered}
E_{i}^{H}=\sum_{i=1}^{n} p_{i} * E_{i}^{H}=\sum_{i=1}^{n} p_{i} *\left\{\frac { \lambda _ { s } } { \lambda _ { s } + \lambda _ { r } } * \frac { 1 } { \eta } * \left[\left(m_{1}+m_{l}\right) g h_{i}+\left(m_{2}+m_{l}\right) *\left(\frac{1}{2} v_{i}^{2}+2 \mu g \Delta s_{i}\right)+m_{2} *\right.\right. \\
\left.\left.\left(\frac{1}{2} v_{i}^{2}+2 \mu g \Delta s_{i}\right)\right]+\frac{\lambda_{r}}{\lambda_{s}+\lambda_{r}} * \frac{1}{\eta} *\left[m_{1} g h_{i}+m_{2} *\left(\frac{1}{2} v_{i}^{2}+2 \mu g \Delta s_{i}\right)+\left(m_{2}+m_{l}\right) *\left(\frac{1}{2} v_{i}^{2}+2 \mu g \Delta s_{i}\right)\right]\right\}(6)
\end{gathered}
$$

Note that this equation takes into account not only the equipment characteristics (i.e. $m_{1}, m_{2}, v_{i}$ and $\eta$ ) but also the rack configuration by means of the terms $h_{i}$, which is a 
function of the number of storage tiers $T$, and $\Delta s_{i}$, which is a function of the number of storage columns.

Given the lack of similar studies, the validation of the energy model has been conducted by considering real case studies. Such validation required to overcome the difficulty in obtaining the data. Indeed, on one hand, material handling providers did not know the actual energy consumption of their customers. On the other hand, the energy consumption data available by users generally referred to the overall warehouse. Two material handling providers has been able to supply us with real case studies, i.e. one with AS/RS and another one with AVS/RS. For each case, we obtained data on the rack configuration (i.e. number of aisles, tiers and columns), the product features, all the information required to apply the model (Table 2), and the average energy consumption per cycle. The accuracy of the analytical models was measured using the absolute

relative error, calculated by the expression $\left(\left|\frac{M-R}{R}\right| \mathrm{x} 100\right)$, where $M$ and $R$ correspond to the estimation obtained from the model and real data, respectively. XXXXXXXXXXXXXXXXXXXXXXXXXXXXXXXXXXXXXXXXXXXXXXXXX Take in Table 2 XXXXXXXXXXXXXXXXXXXXXXXXXXXXXXXXXXXXXXXXXXXXXXXXX

As far as the AS/RS technology is concerned, a distribution centre located in Switzerland has been considered. It is part of the European logistics network of a company operating in the fast-moving consumer goods sector. The validation phase lead to obtain an absolute error equal to $11 \%$ as $M$ and $R$ is $26.3 \mathrm{Wh} /$ cycle and 29.6 Wh/cycle, respectively. As far as the AVS/RS technology is concerned, a distribution centre in United Kingdom has been considered. The company sells goods (mainly household products) through both physical and on-line stores. In this case, the absolute relative error is $6 \%$ as $M$ and $R$ is $1.59 \mathrm{Wh} /$ cycle and $1.5 \mathrm{Wh} /$ cycle, respectively. 
The results suggest that the proposed model allows obtaining an estimation of the energy consumption (average value per cycle), that can be considered representative of real data. Therefore, it can be used to evaluate the energy consumption and environmental impact of the AS/R and AVS/R systems.

\section{Comparison of the automated solutions}

As illustrated in the literature review, the trade-off between economic and environmental dimensions is often present in supply chain contexts. There is no clear evidence to suggest that it also occurs with automated systems and in what way it can affect the selection among different technologies.

The aim of this section is to use the proposed model to investigate whether the selection of automated solutions changes varying the considered dimensions. For this purpose, a comparison between AS/RS and AVS/RS was performed, by:

- First, considering only the economic impact $I^{e c o}$ (Section 5.2)

- Second, considering only the environmental impact $I^{\text {env }}$ (Section 5.3)

- Third, looking at the economic $\left(I^{e c o}\right)$ and environmental $\left(I^{e n v}\right)$ impacts simultaneously (Section 5.4)

The assumptions and the main data used for the analysis are reported in Sections 5.1.

\subsection{Main data and assumptions}

Similarly to previous studies on the comparison of automated systems (Kuo, Krishnamurthy, and Malmborg 2007), a number of scenarios, defined by different combinations of storage and throughput capacity, were evaluated. Storage capacity $(n)$ ranges from 3,000 to 11,000 storage locations in increments of 2,000 , whereas throughput capacity $\left(\lambda=\lambda_{s}+\lambda_{r}\right)$ ranges from 100 to 900 retrievals per hour in 
increments of 200. Therefore, twenty-five scenarios were evaluated. The comparison took into account the characteristics of the technologies examined (e.g. layout, I/O location) and assumptions (e.g. storage and dwell point policies) reported in Section 3. The most common tote size of 400x600x500 mm was assumed. Based on the typical maximum height of an industrial building (i.e. $10 \mathrm{~m}$ ) and a unit clearance height per storage position of $0.7 \mathrm{~m}$, fourteen $T$ values were used in both AS/R and AVS/RS. The maximum length of the rack configuration was $80 \mathrm{~m}$, which corresponds to a maximum number of storage columns of 133. The other main assumptions are as follows:

- Storage throughput capacity $\left(\lambda_{s}\right)$ is equal to the retrieval throughput capacity $\left(\lambda_{r}\right)$

- Acceleration and deceleration warm up are included in the fixed component of the cycle times $\varepsilon^{C}, \varepsilon^{V}$ and $\varepsilon^{L}$

- Warehouse operating time is equal to 3,000 hours per year

In order to compare AS/RS and AVS/RS, the optimal rack configuration must be identified for each scenario. It is defined as the combination of the number of storage tiers $(T)$, aisles $(A)$ and columns $(C)$ that meets the requirements (i.e. storage and throughput capacity and service level) at a minimum economic performance, given the physical constraints. The required service level is met when the target resource utilisation is equal to 0.9 (Kuo, Krishnamurthy, and Malmborg 2007).

As far as the throughput capacity estimation is concerned, cycle time can be obtained by using either simulation or analytical models. Based on the scope of the analysis and the large number of alternatives to be evaluated for each scenario, an analytical approach was chosen and spreadsheets were used as support tools. The widely used model developed by Bozer and White (1984) was adopted to assess AS/RS 
performance. Therefore, the expected time for a single command cycle $\tau^{A S / R S}$ is described by the following equation:

$$
\tau^{A S / R S}=\left(1+\frac{b^{2}}{3}\right) * T_{1}+2 * \varepsilon^{C}
$$

where

$$
\begin{gathered}
T_{1}=\max \left(\frac{L}{v_{y}^{C}}, \frac{H}{v_{z}^{C}}\right) \\
b=\min \left(\frac{L}{v_{y}^{C} * T_{1}}, \frac{H}{v_{z}^{C} * T_{1}}\right)
\end{gathered}
$$

The results obtained by Marchet et al. (2012) were used to assess AVS/RS performance. Therefore, the expected time for a single command cycle performed by a vehicle $\tau_{V}^{A V S / R S}$ is equal to the time required to complete a cycle at the midpoint of the aisle:

$$
\tau_{V}^{A V S / R S}=\frac{L}{2 * v^{V}} * 2+\varepsilon^{V}
$$

Similarly, $\tau_{L}^{A V S / R S}$ is equal to the time required for a cycle to be completed by a lift at the midpoint of its maximum distance travelled:

$$
\tau_{L}^{A V S / R S}=\frac{H}{2 * v^{L}} * 2+\varepsilon^{L}
$$

With reference to the assessment of the economic performance, $I^{e c o}$ can be evaluated in line with previous literature (e.g. Parikh and Meller 2008; Melacini, Perotti, and Tumino 2011) on the basis of the annualised cost as a sum of four cost items: equipment cost (namely cranes for AS/RS and vehicles and lifts for AVS/RS), rack cost, cost of space and cost of energy:

$$
I^{e c o}=\left(A C^{C}+A C^{V} * T+A C^{L}\right) * A+A C^{R} * n+S * C^{S}+E * C^{E}
$$


where:

- $A C^{C}=$ annualised cost of a crane [€/year]

- $A C^{V}=$ annualised cost of a vehicle [€/year]

- $A C^{L}=$ annualised cost of a lift [€/year]

- $A C^{R}=$ annualised cost of a storage rack position [€/year]

- $S=$ space required $\left[\mathrm{m}^{2}\right]$

- $C^{S}=$ cost of space $\left[€ /\left(\mathrm{m}^{2 *}\right.\right.$ year $\left.)\right]$

- $E=$ energy consumption $[\mathrm{kWh} /$ year $]$

- $C^{E}=$ cost of energy $[€ / \mathrm{kWh}]$

It should be noted that $A C^{L}$ and $A C^{V}$ are equal to 0 when assessing $\mathrm{AS} / \mathrm{RS}$, whereas $A C^{C}$ is equal to 0 when assessing AVS/RS. The energy consumption $E$, consisting of the two components $E^{T}$ and $E^{H}$, can be calculated according to the assessment model of the environmental impact provided in Section 4.

The data required to perform the analysis were obtained through interviews with leading material handling providers. Table 3 reports the data for the estimation of the cycle time and energy required for handling operations, whereas the system unit costs are listed in Table 4. As far as the energy $E^{T}$ is concerned, the required temperature in the storage area was taken to be between 0 and $4{ }^{\circ} \mathrm{C}$ and an average external annual temperature of $10{ }^{\circ} \mathrm{C}$ was specified, which is typical for a continental climate. The choice to specify a temperature between 0 and $4{ }^{\circ} \mathrm{C}$ was made in order to evaluate contexts where the energy consumption is significant. Finally, a thermal transmittance of $3.6 \mathrm{~W} /\left(\mathrm{m}^{2 *} \mathrm{~K}\right)$ was assumed. 
XXXXXXXXXXXXXXXXXXXXXXXXXXXXXXXXXXXXXXXXXXXXXXXXX

\subsection{Comparison according to the economic dimension}

Using the data reported in Tables 3 and 4 and assuming 10 years of service and a 10\% interest rate, in accordance with Marchet, Melacini, and Perotti (2011), the optimal rack configuration for both AS/RS and AVS/RS was identified for each scenario (Table 5).

Based on the comparison of the annualised cost for each optimal rack configuration, Figure 3 presents the technology that has the lowest $I^{\text {eco }}$ for each scenario examined.

XXXXXXXXXXXXXXXXXXXXXXXXXXXXXXXXXXXXXXXXXXXXXXXXX

Take in Table 5 and Figure 3

XXXXXXXXXXXXXXXXXXXXXXXXXXXXXXXXXXXXXXXXXXXXXXXXX

The results reported in Figure 3 suggest that from the economic perspective there are well-defined areas of suitability for each of the two systems. Specifically, AVS/RSs seem to be preferable to AS/RS where the required handling capacity is higher with respect to the required number of storage locations. This is due to the aisle throughput capacity of each system. The AVS/RS throughput capacity is much higher than it is for AS/RS. As such, the design of an AS/RS with a low storage capacity and a high throughput capacity means that aisles have a greater number of storage positions than required. The increase in the number of required aisles leads to cost increases due to both the number of cranes and the warehousing area covered. This is shown in Table 5. For example, in the scenarios where the storage capacity is equal to 7,000 storage positions (i.e. scenarios 11 to 15 ) and throughput capacity $\lambda$ is equal to 100 retrievals per hour, the optimal configuration for both systems is the one with 2 aisles. When $\lambda$ 
increases, the AS/RS needs more aisles, unlike the AVS/RS. In case the storage capacity increases as well, AS/RS technology becomes more appropriate than AVS/RS. Note that there are some scenarios presenting little cost savings achievable by selecting the lowest cost alternative. For instance, the potential cost saving related to the selection of AS/RS technology is $1,325 €$ per year in scenario 7. Although the effective boundaries between the suitable areas of the two technologies depend on unit cost values, the ratio of the throughput capacity to the number of storage locations represents a key variable in the technology selection. For high values of this ratio, the AVS/RS technology appears to be the most appropriate. This result supplements the extant literature on the assessment of AVS/RS technology - according to which AVS/RS with tier-to-tier vehicles and pallet as the handling unit are appropriate in contexts with low throughput capacity (Kuo, Krishnamurthy, and Malmborg 2007).

\subsection{Comparison according to the environmental dimension}

As mentioned in Section 4, the environmental impact of automated solutions is given by the product of the energy consumption $(E)$ and factor $(\alpha)$ that converts the energy consumption into indirect $\mathrm{CO}_{2}$ emissions (refer to Equation (1)). In this study, a factor $\alpha$ of $0.363 \mathrm{kgCO}_{2} / \mathrm{kWh}$ was applied. Its calculation has been performed based on the combination of the different methods used to generate energy in a developed country such as Italy.

The Equation (1) has been applied to assess the environmental impact for each optimal configuration reported in Table 5. Results show that indirect emissions of $\mathrm{CO}_{2}$ related to the energy required for handling operations in a year are lower for AVS/RS than AS/RS in all of the scenarios examined (Table 6). For every 1,000 single command cycles, an AVS/RS requires on average $1.07 \mathrm{kWh}$, whereas an AS/RS requires 9.76 $\mathrm{kWh}$, corresponding to 0.39 and $3.54 \mathrm{kgCO}_{2}$ per cycle, respectively. Table 6 also shows 
a wide range in term of energy consumption, and the related $\mathrm{CO}_{2}$ per cycle, among the examined scenarios. This result further highlights the importance to use a model enabling the estimation of the energy consumption per cycle depending on the rack configurations.

When the energy consumed to maintain the required temperature in the storage area $\left(E^{T}\right)$ is considered as well, the AVS/RS technology still has a lower environmental impact: based on all scenarios, the average consumption of the AVS/RS and AS/RS were 8,138 and $12,862 \mathrm{kWh}$ per year, respectively.

\subsection{Comparison according to both economic and environmental dimensions}

According to the results in Sections 5.2 and 5.3, it is clear that in those scenarios in which the AVS/RS technology has lower costs compared to the AS/RS there is no trade-off between economic and environmental perspectives as the evaluation of each dimension separately yield to the selection of the same technology, i.e. AVS/RS. Hence, the trade-off has to be explored only for those scenarios in which the selection of AS/RS allows to cost savings compared to AVS/RS.

As noted in the literature review, a number of approaches have been proposed to evaluate this trade-off in supply chain contexts. In this study, it was decided to assess both the economic and the environmental impacts from a common perspective, i.e. by monetising the environmental impact. The optimal rack configurations reported in Table 5 were assessed by summing the corresponding economic impact $\left(I^{\text {eco }}\right)$ and the monetised value of the environmental impact $\left(I_{M}^{e n v}\right)$. As discussed earlier, there is no widely recognised assessment method in the literature to monetise the environmental impact. For the purposes of this assessment, the economic value of the environmental 
impact was set at two different values taken from the range suggested by Johnson and Hope (2012): $50 € / \mathrm{tCO}_{2}$ and $350 € / \mathrm{tCO}_{2}$.

Considering all scenarios, the applicability areas of the two technologies presented in Figure 3 remain unchanged when the economic value of the environmental impact is equal to $50 € / \mathrm{tCO}_{2}$. However, Figure 4 shows that the suitability area of AVS/RS increases when the economic value of the environmental impact is equal to $350 € / \mathrm{tCO}_{2}$. Therefore, the greater the valorisation of the environmental impact, the greater the trade-off between economic and environmental dimensions is likely to be. Scenario 19 is an example that illustrates how the technology selection changes as a function of both the perspective assumed (i.e. economic perspective or both economic and environmental perspectives) and the value used in the monetisation of the environmental impact. The related results are reported in Figure 5. They confirm that in some cases, when considering the economic dimension only, warehouse designers and logistics managers may select a system that is not actually optimal also from an environmental perspective.

XXXXXXXXXXXXXXXXXXXXXXXXXXXXXXXXXXXXXXXXXXXXXXXXX

Take in Figures 4 and 5

XXXXXXXXXXXXXXXXXXXXXXXXXXXXXXXXXXXXXXXXXXXXXXXXX

In summary, the results demonstrate the suitability of AVS/RS and AS/RS technologies when looking at the economic impact only. In addition, the results show how the monetisation of the environmental impact can influence the technology selection. In particular, the introduction of environmental perspective shows that the suitability of the AVS/RS technology tends to be further corroborated in those cases where a purely economic perspective would already have led to the selection of this technology. When AS/RS technology appears to be preferable from an economic 
viewpoint, this advantage may be offset by the better environmental performance of the AVS/RS technology.

\section{Conclusions}

This paper proposes a model for assessing the energy consumption and the environmental impact of automated warehousing systems. The model for estimating the energy consumption has been validated by using real data provided by material handling providers. The model was applied to incorporate the environmental perspective in the assessment of warehousing automated solutions and to investigate whether this has an impact on their selection. This model is suitable for different warehousing technologies. Its major strength lies in the fact that it links the energy consumption to the rack configuration and equipment characteristics. The explicit modelling of this relationship is of interest when dimensioning and comparing warehousing solutions in the first phase of warehouse design. From a practical viewpoint, the model can be a useful tool for supporting business decision-making processes and can be adopted in the initial design phase when comparing alternative solutions/technologies.

Two specific technologies were studied, namely AS/RS and AVS/RS with the tote as the handling unit. The results are useful to fill some literature gaps. First, this paper provides an initial attempt to face the trade-off between the environmental and economic perspectives - already investigated by previous literature in the broad context of logistics (Quariguasi Frota Neto et al. 2009) - in warehousing contexts. Results indicate that, differently from previous findings on supply chain sustainability, a tradeoff between the environmental and economic perspectives does not always exist in warehousing. Specifically, incorporating the environmental dimension in the comparison of automated technologies does not always lead to select a different 
solution compared to the one obtained from a purely economic comparison. The AVS/RS technology appears to perform better than the AS/RS technology from an environmental perspective due to its greater energy efficiency per cycle. Therefore, in contexts in which the AVS/RS is economically suitable, it is clearly the preferred system and there is no trade-off with respect to the environment. This occurs when the ratio of throughput capacity to the number of storage locations is high (e.g. high handling capacity warehouses such as pharmaceutical distributors, or consolidation buffers before the shipping area). In cases where the AS/RS technology seems to be the most suitable option from the economic viewpoint (i.e. low value of the ratio of throughput capacity to the number of storage locations), the trade-off was examined by monetising the environmental impact.

Second, this analysis confirmed the usefulness of exploring the impact of the sustainable initiatives according to Barber, Beach, and Zolkiewski (2012). Indeed, findings showed that in some scenarios the technology selection shifts from AS/RS to AVS/RS when considering not only the economic but also the environmental impact. However, it should be highlighted that the impact of environmental performance depends on the considered context (e.g. requirements on performance and temperature in the reserve area, product features, sustainability consciousness of the management, and monetised value of the environmental impact).

Third, this research also enriched the extant literature on AVS/RS. According to Kuo, Krishnamurthy, and Malmborg (2007), the AVS/RS with tier-to-tier vehicles and pallet as the handling unit are appropriate in contexts with low values of the ratio the throughput capacity to the number of storage locations. Conversely, results indicated that AVS/RS with tier-captive vehicles and tote as handling unit are suitable for high values of such ratio due to the higher system throughput capacity. 
In summary, this study contributes to the extant literature on the assessment of warehousing automated solutions by reducing the gap towards environmental sustainability in a warehousing context. On the one hand, a more comprehensive evaluation of automated solutions may be performed by introducing the environmental dimension thanks to the proposed model. On the other hand, this study provides new insights on the suitability areas of AVS/RSs. From a practical viewpoint, this study may be a valuable support for companies when selecting their warehousing technology.

However, some limitations of the present research can be identified. First, annualised cost was the key driver used in the economic assessment, whereas other potential factors/constraints related to the decision maker (e.g. target payback time, financial resources) were not taken into account. Second, some parameters that are inputs to the analytical model may have uncertainty associated with them; future simulation-based research could include this uncertainty in the technology selection problem. Third, further development of this research are required towards the concept of "warehouse sustainability". Indeed, the paper focuses on both the economic and environmental dimensions, where this latter refers to the direct $\mathrm{CO}_{2}$ emissions related to energy consumption required for maintaining a target temperature in the warehouse and for handling operations. Potential streams for future research in this arena (i.e. sustainability in warehousing contexts) may go towards the exploration of other aspects in terms of environmental impact (e.g. biodiversity and packaging waste) and the introduction in the analysis of the third dimension of sustainability (i.e. social dimension), according to the Triple Bottom Line (TBL) approach.

\section{Acknowledgement}

This work was supported by material handling suppliers. In particular, TGW Logistics Group is gratefully acknowledged by the authors. 


\section{References}

Baker, P., and M. Canessa. 2009. "Warehouse design: a structured approach." European Journal of Operational Research 193 (2): 425-436.

Ballot, E., and F. Fontane. 2010. "Reducing transportation $\mathrm{CO}_{2}$ emissions through pooling of supply networks: perspectives from a case study in French retail chains." Production Planning \& Control: The Management of Operations 23 (2-3): 105-119.

Barber, K.D., R. Beach, and J. Zolkiewski. 2012. "Environmental sustainability: a value cycle research agenda." Production Planning \& Control: The Management of Operations 21 (6): 640-650.

Bloemhof-Ruuward, J.M., H. Krikke, and L.N. Van Wassenhove. 2004. "OR models for ecoeco closed-loop supply chain optimization." In Reverse logistics: quantitative models for closed-loop supply chains, edited by Dekker, R., M. Fleischmann, and K. Inderfurth, 357379. Berlin: Springer.

Bozer, Y.A., and J.A. White. 1984. "Travel time models for automated storage and retrieval systems." IIE Transactions 16 (4): 329-338.

Chaabane, A., A. Ramudhin, and M. Paquet. 2011. "Designing supply chains with sustainability Considerations." Production Planning \& Control: The Management of Operations 22 (8): 727-741.

Colicchia, C., M. Melacini, and S. Perotti. 2011. "Benchmarking supply chain sustainability: insights from a field study." Benchmarking: An International Journal 18 (5): 705-732.

Dallari, F., G. Marchet, and M. Melacini. 2009. "Design of order picking system." The International Journal of Advanced Manufacturing Technology 42 (1): 1-12.

Ekren, B.Y., and S.S. Heragu. 2012. "Performance comparison of two material handling systems: AVS/RS and CBAS/RS.” International Journal of Production Research 50 (15): 4061-4074.

Fukunari, M., and C.J. Malmborg. 2008. "An efficient cycle time model for autonomous vehicle storage and retrieval systems." International Journal of Production Research 46 (12): 31673184.

Gimenez, C., V. Sierra, and J. Rodon. 2012. "Sustainable operations: their impact on the triple bottom line." International Journal of Production Economics 140 (1): 149-159.

Gua, J., M. Goetschalckx, and L.F. McGinnis. 2010. "Research on warehouse design and performance evaluation: a comprehensive review." European Journal of Operational Research 203 (3): 539-549.

Huppes, G., and M. Ishikawa. 2005. "Eco-efficiency and its terminology." Journal of Industrial Ecology 9 (4): 43-46.

Johnson, L.T., and C. Hope. 2012. "The social cost of carbon in U.S. regulatory impact analyses: an introduction and critique." Journal of Environmental Science 2 (3): 205-221.

Klepper, G., and S. Peterson. 2006. "Marginal abatement cost curves in general equilibrium: the influence of world energy prices." Resource and Energy Economics 28 (1): 1-23.

Kuo, P.H., A. Krishnamurthy, and C.J. Malmborg. 2007. "Design models for unit load storage and retrieval systems using autonomous vehicle technology and resource conserving storage and dwell point policies." Applied Mathematical Modelling 31 (10): 2332-2346.

Langella, I.M., and S. Zanoni. 2011. "Eco-efficiency in logistics: a case study on distribution network design." International Journal of Sustainable Engineering 4 (2): 115-126.

Mahler, D. 2007. "The sustainable supply chain." Supply Chain Management Review 11 (8): 59-60.

Malmborg, C.J. 1996. "An integrated storage system evaluation model.” Applied Mathematical Modelling 20 (5): 359-370. 
Malmborg, C.J. 2002. "Conceptualizing tools for autonomous vehicle storage and retrieval systems." International Journal of Production Research 40 (8): 1807-1822.

Marchet, G., M. Melacini, and S. Perotti. 2011. "A model for design and performance estimation of pick-and-sort order picking systems." Journal of Manufacturing Technology Management, 22 (2): 261-282.

Marchet, G., M. Melacini, S. Perotti, and E. Tappia. 2012. "Analytical model to estimate performances of autonomous vehicle storage and retrieval systems for product totes." International Journal of Production Research 50 (24): 7134-7148.

McKinnon, A., M. Browne, and A. Whiteing. 2012. Green logistics: improving the environmental sustainability of logistics. London: Kogan Page.

Melacini, M., S. Perotti, and A. Tumino. 2011. "Development of a framework for pick-and-pass order picking system design." The International Journal of Advanced Manufacturing Technology 53 (9-12): 841-854.

Meneghetti, A., and L. Monti. 2013. "Sustainable storage assignment and dwell-point policies for automated storage and retrieval systems." Production Planning \& Control: The Management of Operations 24 (6): 511-520.

MHIA (Material Handling Industry of America). 2009. "AS/RS Industry Group Releases Fall 2009 Quarterly Report.” Accessed 3 March 2010.

http://www.mhia.org/news/industry/9141/as-rs-industrygroup-releases-fall-2009-quarterlyreport.

Nordhaus, W. 2011. Estimates of the Social Cost of Carbon: background and resultes from the RICE-2011 model. Cowles Foundation Discussion Papers 1826, Cowles Foundation for Research in Economics, Yale University.

Parikh, P.J., and R.D. Meller. 2008. "Selecting between batch and zone order picking strategies in a distribution center." Transportation Research Part E: Logistics and Transportation Review 44 (5): 696-719.

Pearce, D. 2002. "The Social Cost of Carbon and its policy implications." Oxford Review of Economic Policy 19 (3): 362-384.

Price, R., S. Thornton, and S. Nelson. 2007. The social cost of carbon and the shadow price of carbon: what they are, and how to use them in economic appraisal in the UK. London: Defra.

Quariguasi Frota Neto, J., G. Walther, J. Bloemhof, and T. Spengler. 2009. “A methodology for assessing eco-efficiency in logistics networks." European Journal of Operational Research 193 (3): 670-682.

Ramudhin, A., A. Chaabane, and M. Paquet. 2009. "On the design of sustainable, green supply chains." In Proceedings of the 39th International Conference on Computers \& Industrial Engineering. Troyes, France.

Rizzo, J. 2006. "Logistics distribution \& warehousing 2006: green building: a new priority." http://www.areadevelopment.com/AssetManagement/dec07/greenBuilding.shtml.

Roodbergen, K.J., and I.F.A. Vis. 2009. "A survey of literature on automated storage and retrieval systems." European Journal of Operation Research 194 (2): 343-362.

Seuring, S., and M. Müller. 2008. "From a literature review to a conceptual framework for sustainable supply chain management." Journal of Cleaner Production 16 (15): 1699-1710.

Tan, K-S, M.D. Ahmed, and D. Sundaram. 2010. "Sustainable enterprise modelling and simulation in a warehousing context." Business Process Management Journal 16 (5): 871886.

UNI/TS 11300, 2010. Norme tecniche per la stima della prestazione energetica degli edifici. Ente Nazionale Italiano di Unificazione (UNI), Milano, Italy. 
Zhang, L., A. Krishnamurthy, C.J. Malmborg, and S.S. Heragu. 2009. "Variance-based approximations of transaction waiting times in autonomous vehicle storage and retrieval systems.” European Journal of Industrial Engineering 3 (2): 146-169. 


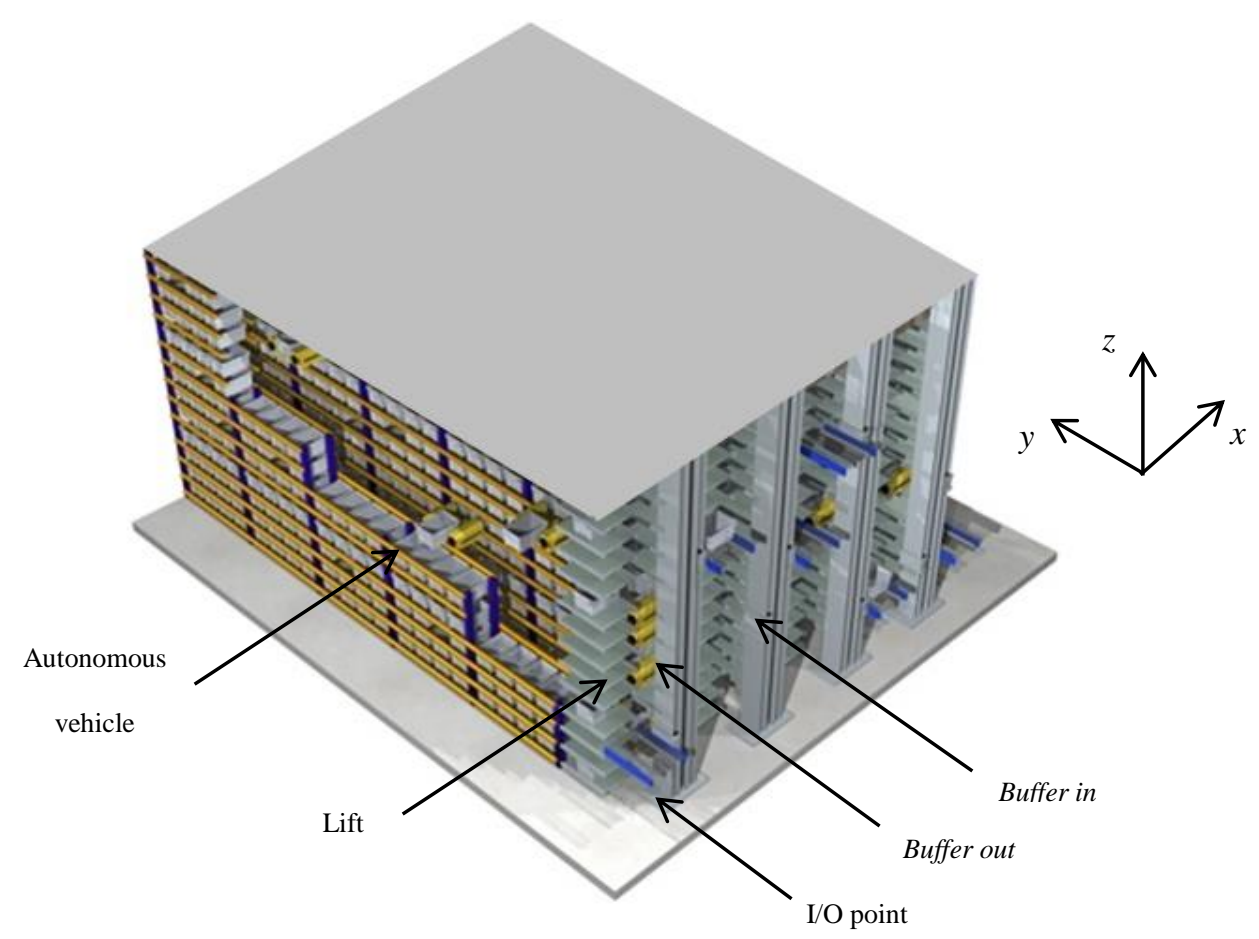

Figure 1. AVS/RS with tier-captive vehicles and tote as handling unit (derived from Marchet et al. 2012).

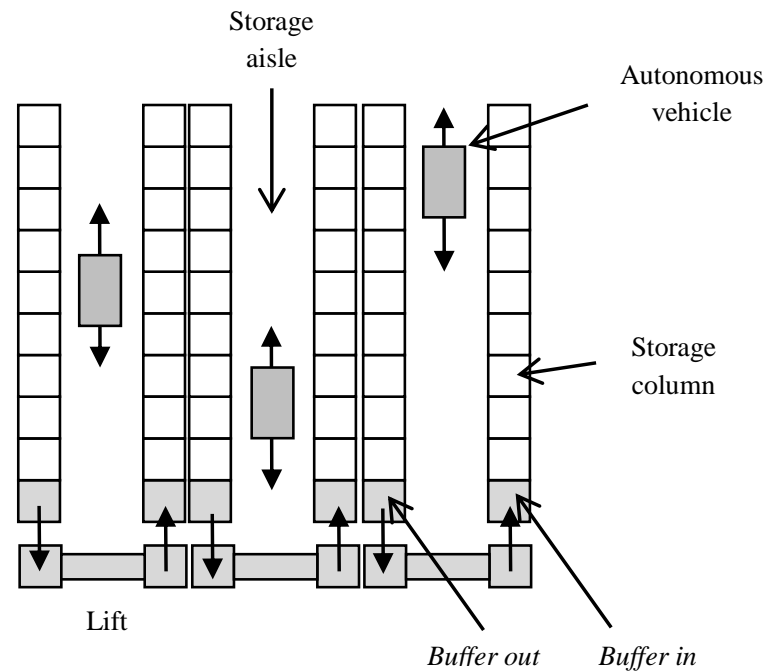

Figure 2. Single tier of an AVS/RS with tier-captive vehicles and tote as handling unit (derived from Marchet et al. 2012). 


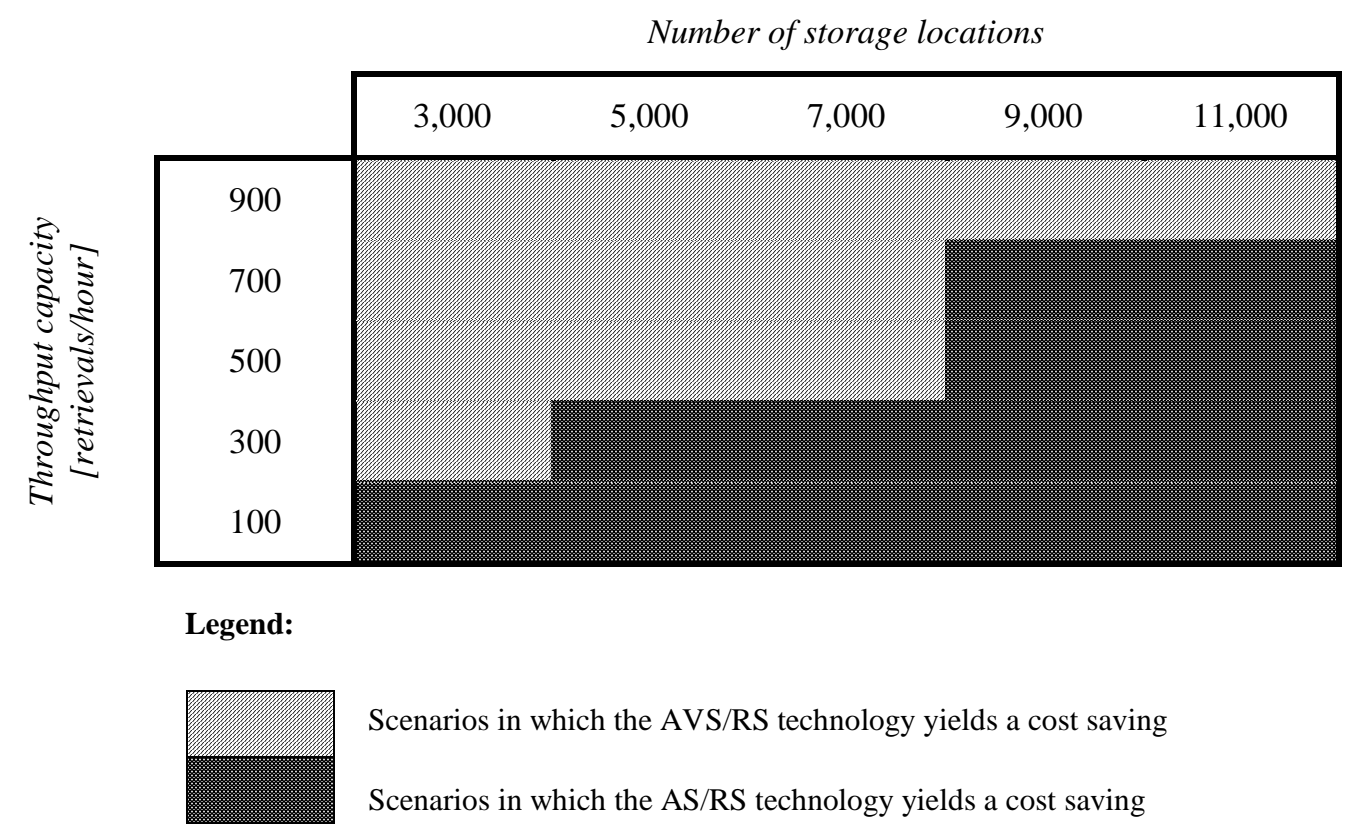

Figure 3. Illustration of the technology that yields a cost saving for each scenario from a purely economic perspective.

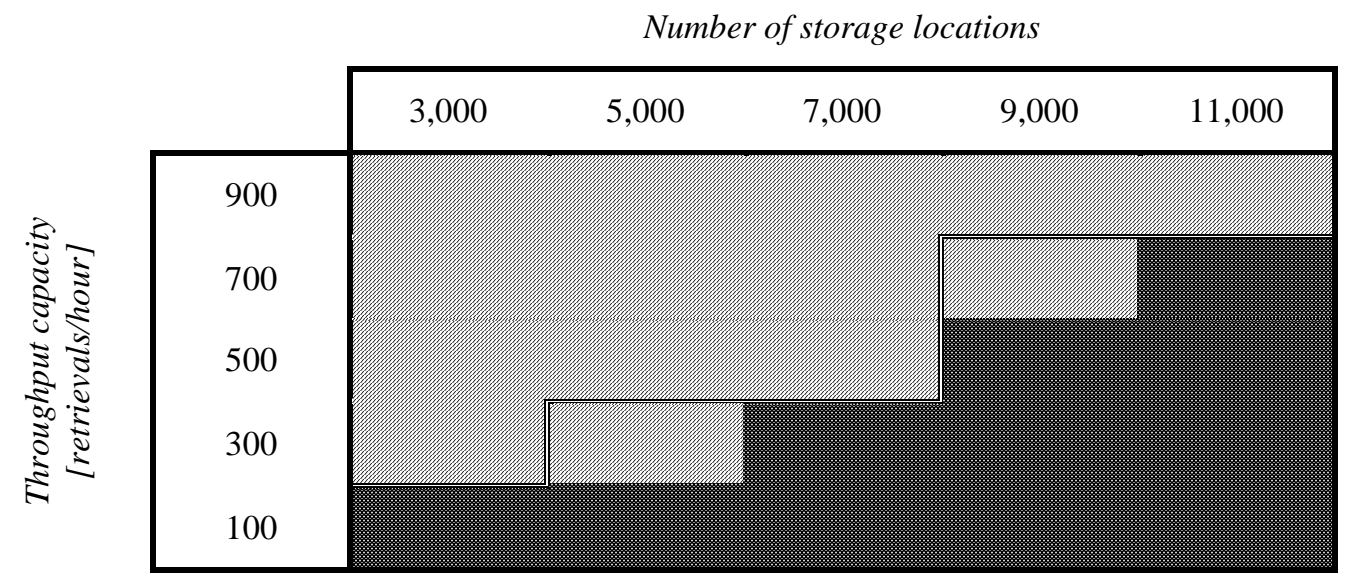

Legend:

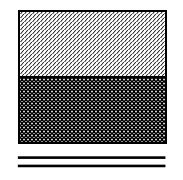

Scenarios in which the AVS/RS technology yields a cost saving

Scenarios in which the AS/RS technology yields a cost saving

Boundary of the adoption area according to a purely economic perspective

Figure 4. Illustration of the technology that yields a cost saving for each scenario based on a combined economic and environmental perspective, assuming $350 € /$ tonne of $\mathrm{CO}_{2}$ to monetise the environmental impact. 


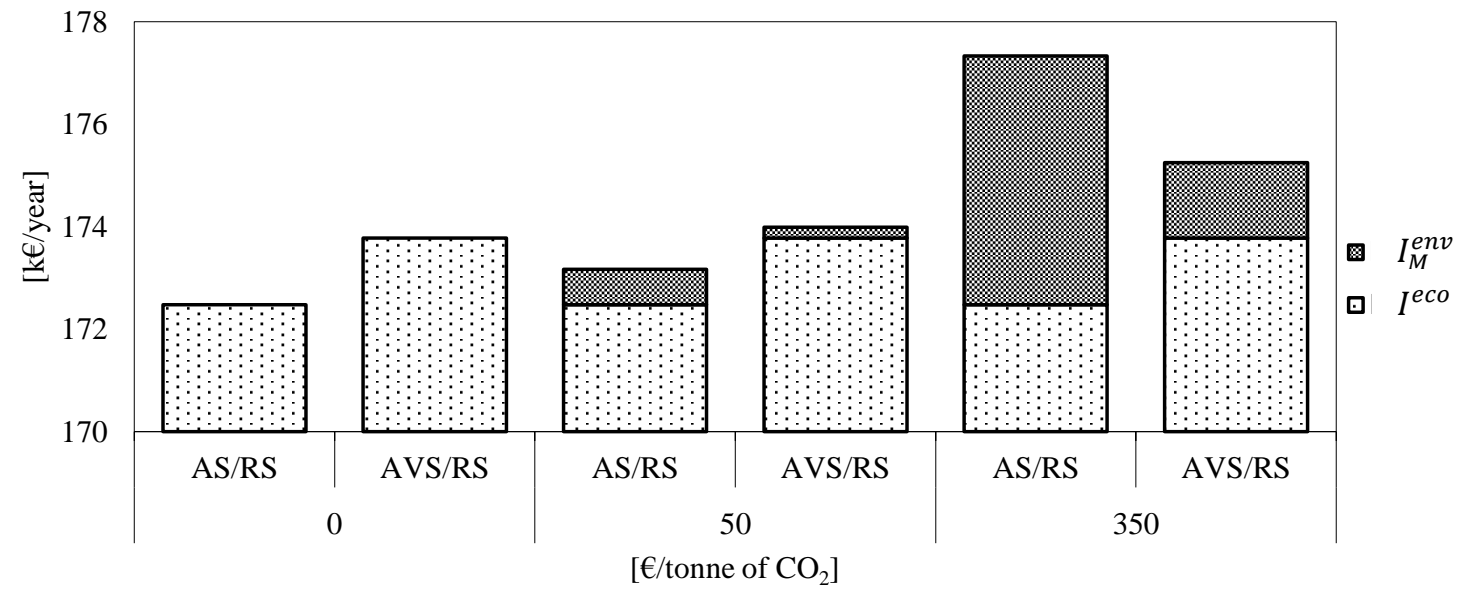

Figure 5. Illustration of the trade-off between economic and environmental performance for the scenario involving 700 retrievals/hour and 9,000 storage locations. 
Table 1. Main notations.

\begin{tabular}{ll}
\hline Notations & Description \\
\hline$A$ & No. of storage aisles \\
$T$ & No. of storage tiers \\
$C$ & No. of storage columns on each side of a storage aisle \\
$N$ & Total number of storage positions \\
$L, H$ & Maximum travel distance to be covered along the $y$ - and $z$-axes [m] \\
$\lambda_{s}, \lambda_{r}$ & System storage and retrieval throughput capacity [cycles/h] \\
$\mu_{w}, \mu_{h}$ & Unit width and height of clearance per storage position both in AS/RS and AVS/RS [m] \\
$v_{z}^{C}, v_{y}^{C}$ & Maximum crane velocity along the $z$ - and $y$-axes [m/s] \\
$v^{V}, v^{L}$ & Maximum vehicle and lift velocity [m/s] \\
$a_{z}^{C}, a_{y}^{C}$ & Crane acceleration/deceleration along the $z$ - and $y$-axes respectively [m/s ${ }^{2}$ ] \\
$a^{V}, a^{L}$ & Vehicle and lift acceleration/deceleration [m/s ${ }^{2}$ ] \\
$\varepsilon^{C}$ & Fixed part of the crane cycle time (i.e. braking delay times, time allowance for charging \\
$\varepsilon^{V}, \varepsilon^{L}$ & $\begin{array}{l}\text { Fixd discharging load and fork cycle time) [s] } \\
\text { charging and discharging load and fork cycle time) [s] }\end{array}$ \\
\hline
\end{tabular}

Table 2. Data used in the validation.

\begin{tabular}{llll}
\hline Variable & Unit of measure & Values in the AS/RS case & Values in the AVS/RS case \\
\hline$A$ & No. of storage aisles & 29 & 8 \\
$T$ & No. of storage tiers & 20 & 20 \\
& $\begin{array}{l}\text { No. of storage } \\
\text { columns on each }\end{array}$ & 150 & 34 \\
$C$ & side of an aisle & & \\
& $\mathrm{m}$ & 0.6 & 0.6 \\
$\mu_{\boldsymbol{w}}$ & $\mathrm{m}$ & 0.7 & 0.7 \\
$\mu_{h}$ & $\mathrm{~kg}$ & 100 & 50 \\
$m_{l}$ & $\mathrm{~kg}$ & 500 & - \\
$m_{1}^{A S / R S}$ & $\mathrm{~kg}$ & - & 50 \\
$m_{1}^{A V S / R S}$ & $\mathrm{~kg}$ & 2,000 & - \\
$m_{2}^{A S / R S}$ & $\mathrm{~kg}$ & - & 80 \\
$m_{2}^{A V S / R S}$ & $\mathrm{~m} / \mathrm{s}$ & 6 & - \\
$v_{z}^{C}$ & $\mathrm{~m} / \mathrm{s}$ & 3 & - \\
$v_{y}^{C}$ & $\mathrm{~m} / \mathrm{s}$ & - & 1.5 \\
$v^{V}$ & $\mathrm{~m} / \mathrm{s}$ & - & 4 \\
$v^{L}$ & $\mathrm{~m} / \mathrm{s}^{2}$ & 3 & - \\
$a_{z}^{C}$ & $\mathrm{~m} / \mathrm{s}^{2}$ & 3.5 & - \\
$a_{y}^{C}$ & $\mathrm{~m} / \mathrm{s}^{2}$ & - & 1 \\
$a^{V}$ & $\mathrm{~m} / \mathrm{s}^{2}$ & - & 7 \\
$a^{L}$ & $\mathrm{~s}$ & 4 & - \\
$\varepsilon^{C}$ & $\mathrm{~s}$ & - & 5 \\
$\varepsilon^{V}$ & $\mathrm{~s}$ & & 3 \\
$\varepsilon^{L}$ & & - & \\
\hline
\end{tabular}


Table 3. Data used in the comparison.

\begin{tabular}{lll}
\hline Variable & Unit of measure & Values \\
\hline$\mu_{w}$ & $\mathrm{~m}$ & 0.6 \\
$\mu_{h}$ & $\mathrm{~m}$ & 0.7 \\
$m_{l}$ & $\mathrm{~kg}$ & 75 \\
$m_{1}^{A S / R S}$ & $\mathrm{~kg}$ & 300 \\
$m_{1}^{A V S / R S}$ & $\mathrm{~kg}$ & 50 \\
$m_{2}^{A S / R S}$ & $\mathrm{~kg}$ & 1,000 \\
$m_{2}^{A V S / R S}$ & $\mathrm{~kg}$ & 100 \\
$v_{z}^{C}$ & $\mathrm{~m} / \mathrm{s}$ & 3 \\
$v_{y}^{C}$ & $\mathrm{~m} / \mathrm{s}$ & 5 \\
$v^{V}$ & $\mathrm{~m} / \mathrm{s}$ & 1.5 \\
$v^{L}$ & $\mathrm{~m} / \mathrm{s}$ & 4 \\
$a_{z}^{C}$ & $\mathrm{~m} / \mathrm{s}^{2}$ & 1.2 \\
$a_{y}^{C}$ & $\mathrm{~m} / \mathrm{s}^{2}$ & 1 \\
$a^{V}$ & $\mathrm{~m} / \mathrm{s}^{2}$ & 1 \\
$a^{L}$ & $\mathrm{~m} / \mathrm{s}^{2}$ & 7 \\
$\varepsilon^{C}$ & $\mathrm{~s}$ & 4 \\
$\varepsilon^{V}$ & $\mathrm{~s}$ & 5 \\
$\varepsilon^{L}$ & $\mathrm{~s}$ & 2 \\
\hline
\end{tabular}

Table 4. Unit costs.

\begin{tabular}{lll}
\hline Cost item & Unit of measure & Value \\
\hline Crane & $€ /$ unit & 150,000 \\
Vehicle & $€ /$ unit & 15,000 \\
Lift & $€ /$ unit & 50,000 \\
Storage position - AS/RS & $€ /$ unit & 15 \\
Storage position - AVS/RS & $€ /$ unit & 25 \\
Area & $€ /\left(\mathrm{m}^{2 *}\right.$ year $)$ & 50 \\
Energy & $€ / \mathrm{kWh}$ & 0.2 \\
\hline
\end{tabular}


Table 5. Optimal rack configuration data for each scenario.

\begin{tabular}{|c|c|c|c|c|c|c|c|c|}
\hline \multirow[b]{2}{*}{ Scenario } & \multirow{2}{*}{$\begin{array}{l}\text { No. of } \\
\text { storage } \\
\text { locations } \\
(n)\end{array}$} & \multirow{2}{*}{$\begin{array}{l}\text { Throughput } \\
\text { capacity }(\lambda) \\
\text { [retrievals } \\
\text { per hour] }\end{array}$} & \multicolumn{3}{|c|}{$\begin{array}{l}\text { Optimal AS/RS rack } \\
\text { configuration }\end{array}$} & \multicolumn{3}{|c|}{$\begin{array}{l}\text { Optimal AVS/RS rack } \\
\text { configuration }\end{array}$} \\
\hline & & & $\begin{array}{l}\text { No. of } \\
\text { aisles } \\
(A)\end{array}$ & $\begin{array}{l}\text { No. of } \\
\text { tiers } \\
(T)\end{array}$ & $\begin{array}{l}\text { No. of } \\
\text { columns } \\
(C)\end{array}$ & $\begin{array}{l}\text { No. of } \\
\text { aisles } \\
(A)\end{array}$ & $\begin{array}{l}\text { No. of } \\
\text { tiers } \\
(T)\end{array}$ & $\begin{array}{l}\text { No. of } \\
\text { columns } \\
(C)\end{array}$ \\
\hline 1 & 3,000 & 100 & 1 & 14 & 108 & 1 & 12 & 125 \\
\hline 2 & 3,000 & 300 & 2 & 14 & 54 & 1 & 12 & 125 \\
\hline 3 & 3,000 & 500 & 3 & 14 & 36 & 1 & 12 & 125 \\
\hline 4 & 3,000 & 700 & 4 & 14 & 27 & 2 & 7 & 108 \\
\hline 5 & 3,000 & 900 & 5 & 14 & 22 & 2 & 8 & 94 \\
\hline 6 & 5,000 & 100 & 2 & 14 & 90 & 2 & 10 & 125 \\
\hline 7 & 5,000 & 300 & 3 & 14 & 60 & 2 & 10 & 125 \\
\hline 8 & 5,000 & 500 & 4 & 14 & 45 & 2 & 10 & 125 \\
\hline 9 & 5,000 & 700 & 4 & 14 & 45 & 2 & 10 & 125 \\
\hline 10 & 5,000 & 900 & 5 & 14 & 36 & 2 & 10 & 125 \\
\hline 11 & 7,000 & 100 & 2 & 14 & 125 & 2 & 14 & 125 \\
\hline 12 & 7,000 & 300 & 3 & 14 & 84 & 2 & 14 & 125 \\
\hline 13 & 7,000 & 500 & 4 & 14 & 63 & 2 & 14 & 125 \\
\hline 14 & 7,000 & 700 & 5 & 14 & 50 & 2 & 14 & 125 \\
\hline 15 & 7,000 & 900 & 6 & 14 & 42 & 2 & 14 & 125 \\
\hline 16 & 9,000 & 100 & 3 & 14 & 108 & 3 & 12 & 125 \\
\hline 17 & 9,000 & 300 & 3 & 14 & 108 & 3 & 12 & 125 \\
\hline 18 & 9,000 & 500 & 4 & 14 & 81 & 3 & 12 & 125 \\
\hline 19 & 9,000 & 700 & 5 & 14 & 65 & 3 & 12 & 125 \\
\hline 20 & 9,000 & 900 & 6 & 14 & 54 & 3 & 12 & 125 \\
\hline 21 & 11,000 & 100 & 3 & 14 & 131 & 3 & 14 & 131 \\
\hline 22 & 11,000 & 300 & 3 & 14 & 131 & 3 & 14 & 131 \\
\hline 23 & 11,000 & 500 & 4 & 14 & 99 & 3 & 14 & 131 \\
\hline 24 & 11,000 & 700 & 5 & 14 & 79 & 3 & 14 & 131 \\
\hline 25 & 11,000 & 900 & 6 & 14 & 66 & 3 & 14 & 131 \\
\hline
\end{tabular}


Table 6. Environmental impact for the optimal AS/RS and AVS/RS rack configuration in each scenario.

\begin{tabular}{lllll}
\hline Scenario & $\begin{array}{l}\text { Number of } \\
\text { storage } \\
\text { locations }(n)\end{array}$ & $\begin{array}{l}\text { Throughput } \\
\text { capacity }(\lambda) \\
\text { [retrievals per hour }]\end{array}$ & $\begin{array}{l}\text { AS/RS environmental } \\
\text { impact } \\
{\left[\mathrm{kgCO}_{2} / 1,000 \text { cycles }\right]}\end{array}$ & $\begin{array}{l}\text { AVS/RS environmental } \\
\text { impact } \\
{\left[\mathrm{kgCO}_{2} / 1,000 \text { cycles }\right]}\end{array}$ \\
\hline 1 & 3,000 & 100 & 3.89 & 0.43 \\
2 & 3,000 & 300 & 3.51 & 0.43 \\
3 & 3,000 & 500 & 2.97 & 0.43 \\
4 & 3,000 & 700 & 2.64 & 0.26 \\
5 & 3,000 & 900 & 2.45 & 0.29 \\
\hline 6 & 5,000 & 100 & 3.98 & 0.36 \\
7 & 5,000 & 300 & 3.63 & 0.36 \\
8 & 5,000 & 500 & 3.28 & 0.36 \\
9 & 5,000 & 700 & 3.28 & 0.36 \\
10 & 5,000 & 900 & 2.97 & 0.36 \\
\hline 11 & 7,000 & 100 & 3.75 & 0.40 \\
12 & 7,000 & 300 & 3.93 & 0.40 \\
13 & 7,000 & 500 & 3.68 & 0.40 \\
14 & 7,000 & 700 & 3.42 & 0.40 \\
15 & 7,000 & 900 & 3.18 & 0.40 \\
\hline 16 & 9,000 & 100 & 3.89 & 0.43 \\
17 & 9,000 & 300 & 3.89 & 0.43 \\
18 & 9,000 & 500 & 3.90 & 0.43 \\
19 & 9,000 & 700 & 3.71 & 0.43 \\
20 & 9,000 & 900 & 3.51 & 0.43 \\
\hline 21 & 11,000 & 100 & 3.74 & 0.40 \\
22 & 11,000 & 300 & 3.74 & 0.40 \\
23 & 11,000 & 500 & 4.05 & 0.40 \\
24 & 11,000 & 700 & 3.88 & 0.40 \\
25 & 11,000 & 900 & 3.72 & \\
\hline & & & & \\
\hline
\end{tabular}

